\title{
The Development of Key Technologies in Applications of Vessels Connected to the Internet
}

\author{
Zhe Tian $^{1}$ (1) , Fushun Liu ${ }^{1, *}$, Zhixiong $\mathrm{Li}^{2,3}$ (D), Reza Malekian ${ }^{4, *}$ (D) and Yingchun Xie ${ }^{1}$ \\ 1 College of Engineering, Ocean University of China, Qingdao 266100, China; tianzhe@ouc.edu.cn (Z.T.); \\ xieyingchun01@163.com (Y.X.) \\ 2 School of Mechatronics Engineering, China University of Mining \& Technology, Xuzhou 221106, China; \\ zhixiong.li@ieee.org \\ 3 School of Mechanical Engineering, Iowa State University, Ames, IA 50011, USA \\ 4 Department of Electrical, Electronic \& Computer Engineering, University of Pretoria, \\ Pretoria 0002, South Africa \\ * Correspondence: percyliu@ouc.edu.cn (F.L.); reza.malekian@ieee.org (R.M.); \\ Tel.: +86-137-9248-7135 (F.L.); +27-124-204-305 (R.M.)
}

Received: 6 August 2017; Accepted: 18 September 2017; Published: 1 October 2017

\begin{abstract}
With the development of science and technology, traffic perception, communication, information processing, artificial intelligence and the shipping information system have become important in supporting the realization of intelligent shipping transportation. Against this background, the Internet of Vessels (IoV) is proposed to integrate all these advanced technologies into a platform to meet the requirements of international and regional transportations. The purpose of this paper is to analyze how to benefit from the Internet of Vessels to improve the efficiency and safety of shipping, and promote the development of world transportation. In this paper, the IoV is introduced and its main architectures are outlined. Furthermore, the characteristics of the Internet of Vessels are described. Several important applications that illustrate the interaction of the Internet of Vessels' components are proposed. Due to the development of the Internet of Vessels still being in its primary stage, challenges and prospects are identified and addressed. Finally, the main conclusions are drawn and future research priorities are provided for reference and as professional suggestions for future researchers in this field.
\end{abstract}

Keywords: ship intelligence; big data; internet of vessels; information fusion

\section{Introduction}

It is well known that over $70 \%$ of the earth's surface is covered by ocean, which leads to the result that the waterway has to undertake more than $80 \%$ of the world's trade transportation, including the seaborne trades and the inland river navigation. In recent years, with the development of technologies such as sensing, telecommunications, computers, information, automation and smart control, a new technological revolution called internet of vessels (IoV) is proposed to meet the requirements of international and regional transportation. The IoV is defined as a network of smart interconnected vessels and the shore facilities with a series of digital entities [1]. Based on the global positioning system and wireless communication technology, the ship intelligent information service could use electronic sensing devices to exchange information on the internet and realize the extraction, supervision and utilization of each nodes' properties and dynamic information on the network platforms. The information is available in real time by way of communication (human to vessel, vessel to vessel, vessel to cargo, vessel to coast) [2]. As a result, the IoV has the ability to navigate, communicate and offer security protection to provide a more intelligent and safer navigating environment. 
In this paper, an in-depth view of the IoV is provided. Due to the dramatic increase in world trade, new challenges with efficiency, safety and energy saving have emerged in the transportation system. As one of the most important transportation modes, the shipping industry is trying to solve the key problem of improving the intelligence level of the vessels and their operating network. The emerging IoV technologies could achieve this goal with precision management, comprehensive service and humanized experience. The purpose of this paper is to analyze how to benefit from the Internet of Vessels to create a more intelligent and safer navigating environment.

This paper is structured as follows: Section 2 introduces the definition of the IoV and its architecture from different technical perspectives; the characteristics of the internet of vessels are discussed in Section 3; in Section 4 the application and benefits of the internet of vessels are described; in Section 5 the challenges and prospects of the emerging technologies are outlined; and finally, a conclusion is drawn in Section 6.

\section{Internet of Vessels Architecture}

As a product in the global information era, the Internet of Things is a very important component of information technology. Based on the frame of the IoT (Internet of Things) technology, the ship's specific characteristics and the application environment, the internet of vessels is proposed. In 1998, the USA developed a kind of integrated service system called the Intelligent Website System (IWS) which contained a ship Automatic Identification System (AIS), data exchange system, navigation system and information network system [3]. At the beginning of the 21st century, the European Union conducted integrated information services for the inland waterway, the River Information Services (RIS), which is a prototype of the IoV [4-6]. The existing structure of the IoV is shown in Figure 1, which consists of vessels, island base stations, a climatic observation system, communication satellites, buoys, wireless transmitting and receiving devices, and the command system.

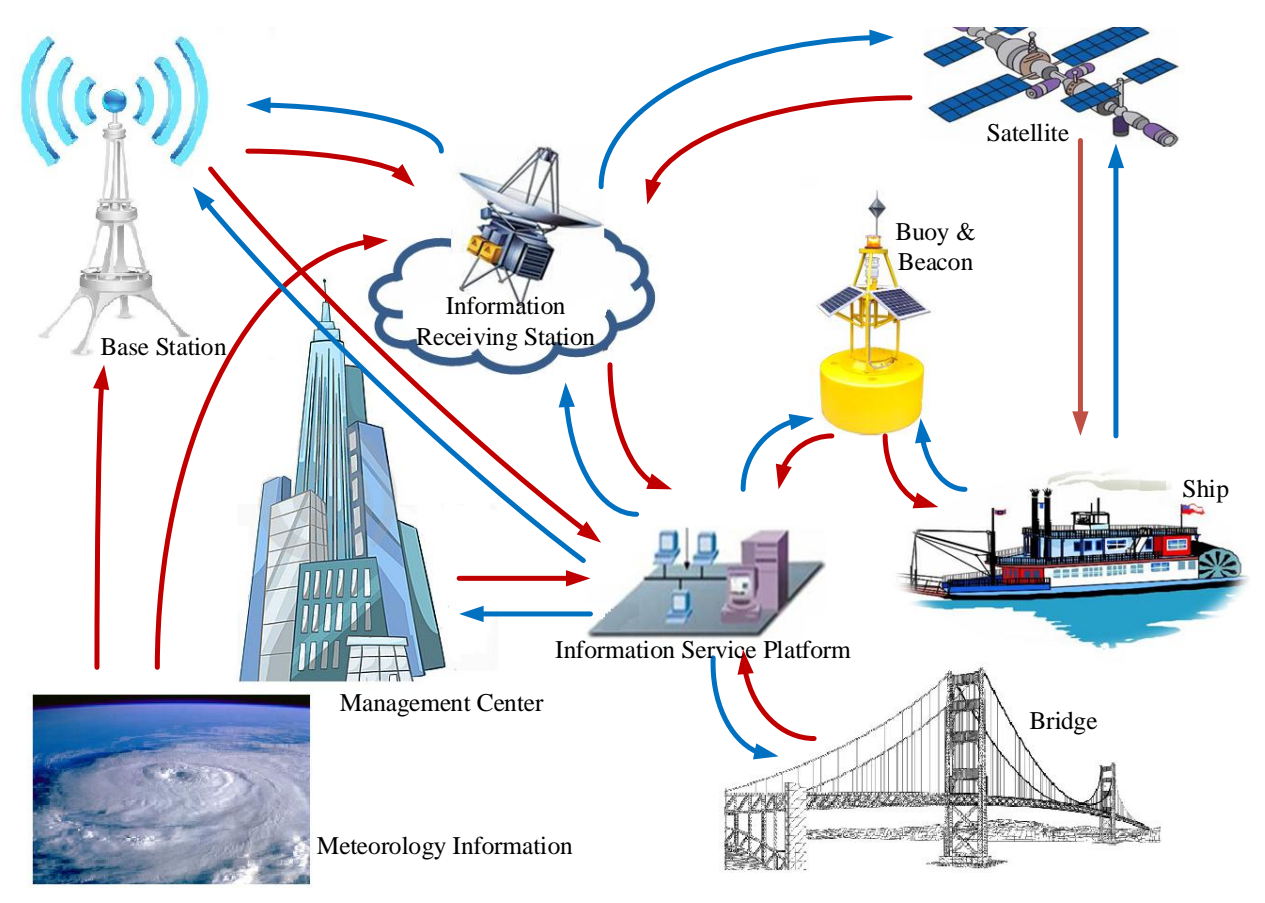

Figure 1. The structure of the IoV.

\subsection{The Classification of the IoV}

At present, there is no clear classification for the IoV. Qin [1] classified the IoV on the basis of the geography and shipping purpose which is shown in Figure 2. 


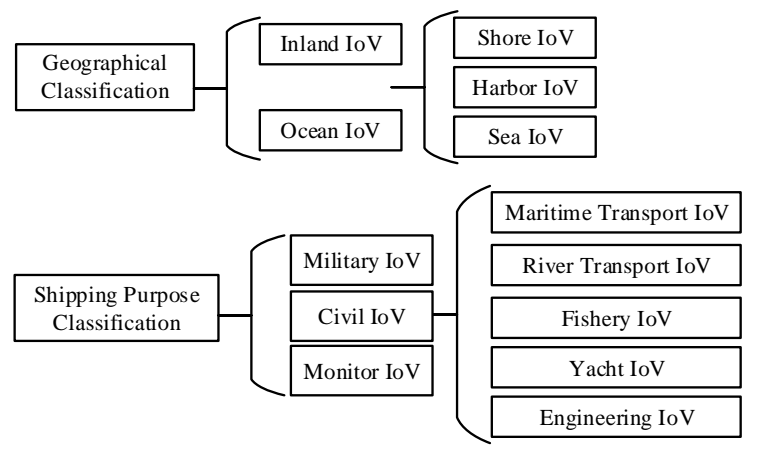

Figure 2. The classification of the IoV based on the geography and ship's purpose.

The RIS system is a combination of various information systems such as the ship declaration information system IBIS/GINA (Belgium), the regional and transnational shipping information system BICS (The Netherland), the inland waterway shipping information network system VNF2000 (France) and the cargo declaration information system MIB/MOVES (Germany). After years of development in the European region, the RIS system has obtained a series of completed system architectures $[7,8]$. The architecture of the RIS system is shown in Figure 3 [9,10]. The RIS system aims at building a cross-regional, cross-system, cross-governmental system with integrated resources. The RIS unifies the technical and legal standards, and realizes the collaboration and normalization of the inland ship service. Covering all the key logistics elements of inland transportation, the RIS which serves the modern shipping logistics industry and supports multimodal transportation is a milestone in the development of building the inland waterway information system in Europe [11]. The success of the RIS system provides valuable experience for the Internet of Vessels construction.

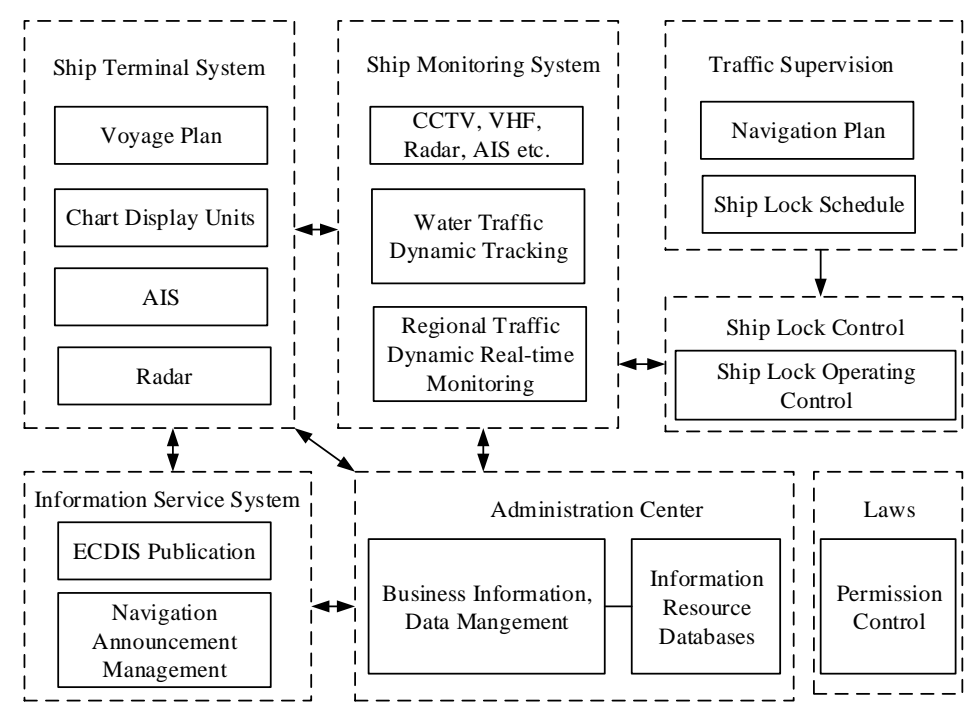

Figure 3. The architectures of the RIS system.

Similarly, Dong [12] and Dai [13] proposed the overall architecture of the IoV on the technology level to meet the three major characteristics of the IoT, which were instrumented, interconnected and intelligent. Dong [12] presented a novel and comprehensive architecture of the IoV system for future reference. The overall architecture of the IoV proposed by Dong [12] is shown in Figure 4, which contains a standard specification guarantee system and an information security assurance system with four layers of perception, network, application and exhibition.

For the perception layer in Figure 4, based on the state intelligence of the shipping infrastructure and ship operating environment as well as the ship visual detection and identification technology, the 
perception layer technology system takes advantage of the shore-based sensors and shipborne sensors including radar, RFID, AIS, GPS and CCTV to construct the technical architecture model of the target in different scenarios. The networks layer is the basic platform to realize the information exchange of the IoV system. According to the multi-network fusion technology [14-16], it aims at high data transmission reliability and high-security to guarantee the perceptive data connection is smooth and successful. Based on the networks layer, the data resource layer is in charge of data collection, saving and management from the perception layer. At the same time, it is the foundation of the information service for the application layer. The application layer is the core of the IoV system. In this layer, various application systems are developed to call resources from the data resource layer to analyze, calculate and process. As a result, the applications are used to meet the demands of various customers. The exhibition layer is the service window with which the IoV system communicates with customers directly. In the exhibition layer, the IoV system provides a virtual service platform for customers according to mobiles, radios, websites, VHF, AIS, intelligent terminals and so on. It satisfies both the requirement of the releasing information service and the access function from the outside information providers, which is quite useful for improving the service level of maritime transportation.

More details will be described in the components of each layer in Figure 4.

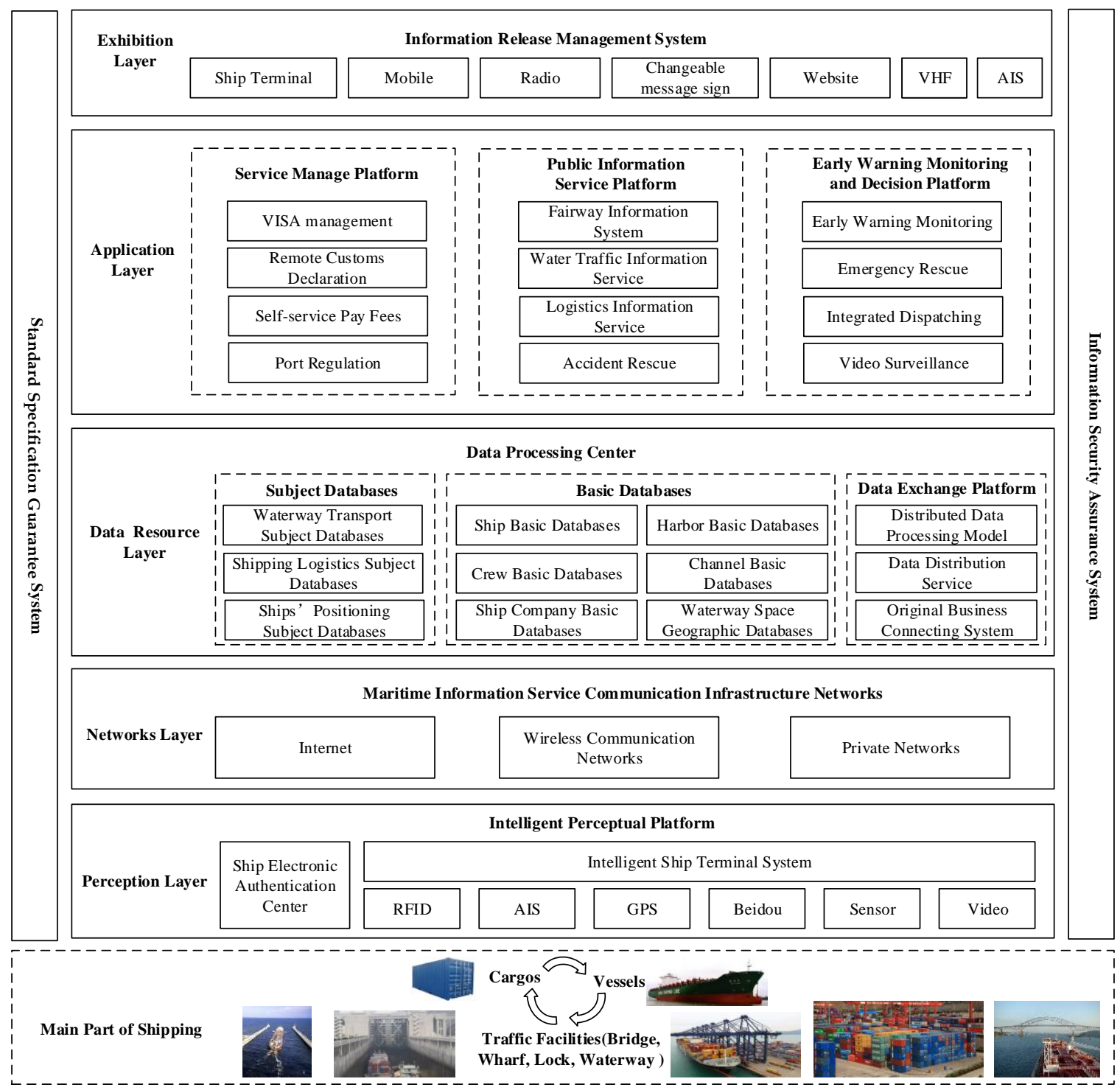

Figure 4. The overall architecture of IoV. 


\subsection{The Technical Architecture of the Perception Layer}

As one of the most important fundaments in the IoV system, the intelligent perception platform contains the ship electronic authentication center, the intelligent ship terminal, sensors and image detection, and the identification system. Based on the collection of the ship positioning data, operating data, waterway traffic data and emergency data, the perception layer can provide the data support for the upper layer [17]. The function of the ship electronic authentication center is to identify that the operating ships are legitimate. The intelligent ship terminal is the core of the perception layer. The RFID module, GPS/Beidou module and sensor module are integrated in this part to provide data (cargos, ships, ports) collection and exchange with the shore-based facilities and servers. The technical architecture of this layer is shown in Figure 5.

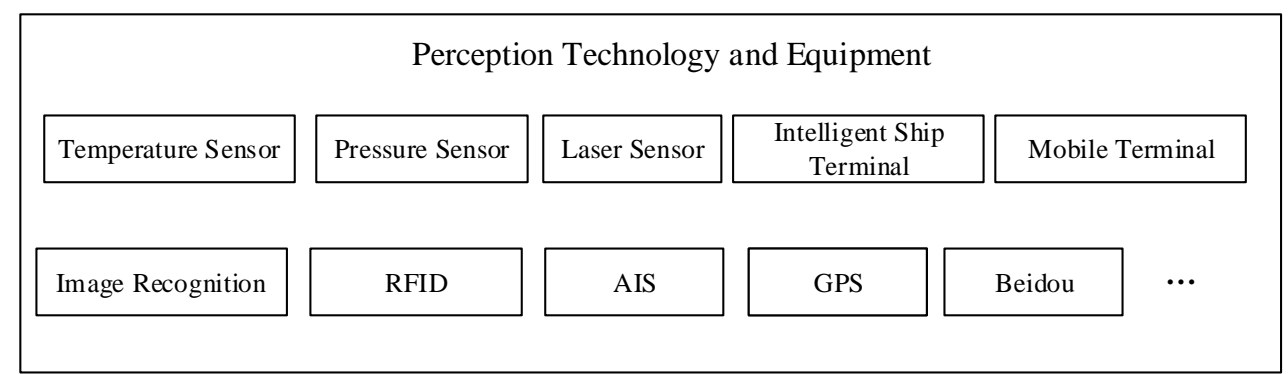

(a)

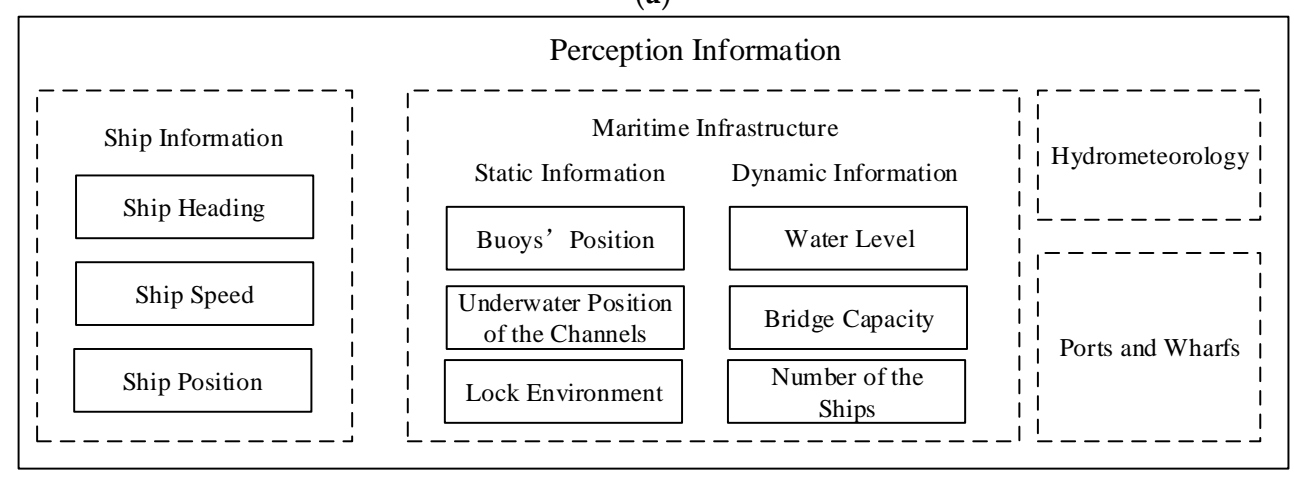

(b)

Figure 5. The technical architecture of the perception layer. (a) The perception technology and equipment of the perception layer; (b) The perception information of the perception layer.

In Figure 5, it can be seen that the IoV system could obtain the dynamic perception of the maritime information in real-time in a variety of ways. The object of the dynamic perception covers a wide range of roles including waterways, ports, ships, intersections, inland river-way service centers, refuse collection points, ship locks, bridges, fleets and marine service stations. Every perceiving subject has its own perceiving object and contents. For instance, in the Yangtze river, in order to realize the construction of the IoV system, the state of the navigation environment which includes the channels, hydrometeorology and navigation structures should be perceived. Generally, the buoys identify the boundary of the channels and provide the information on traffic flow direction, channel obstructions, channel width and channel depth. Therefore, the GNSS (Global Navigation Satellite System) is installed in the buoys to monitor their position. At the same time, Dai [13] proposed a kind of estimation method to calculate the floating threshold of buoys in the safety area. The use of a high precision positioning method for buoys helps the management center control the information on the channels at any time. Besides, with the feature image learning technology based on the BP (back propagation) neural network and the night visibility monitoring technology based on the dual differential luminance algorithm, it could distinguish the trend of bad weather and provide the meteorology information to the management center. Similarly, lots of different sensors are mounted on the navigation structures to 
collect the information about the ships, hydrometeorology and channels. Thus, based on the perception technology, the specific perceiving objects and contents are listed in Table 1 [13].

Table 1. Details of perception information including the perceiving object and its contents.

\begin{tabular}{|c|c|c|c|}
\hline Perceiving Object & Perceiving Contents & Perceiving Object & Perceiving Contents \\
\hline \multirow{8}{*}{ Channels } & Water-level Information & \multirow{8}{*}{ Ship Locks } & Open Time of Locks \\
\hline & Real-time Meteorological Information & & Cargos Information \\
\hline & Regional Geographic Information & & Traffic Conditions \\
\hline & Channel Obstruction & & Time Span Arriving Sites \\
\hline & River and Canal Status & & Estimated Time of Departure \\
\hline & Auxiliary Facilities Status & & Estimated Time of Arrival \\
\hline & Channel Congestion Information & & Short-term Planning of Locks \\
\hline & Emergency Accident Information & & Long-term Planning of Ships \\
\hline \multirow{5}{*}{ Ports } & Docking Information & Intersections & Direction of Channels \\
\hline & Ships' Waiting Spot & Inland Riverway & Type of Service \\
\hline & Cargos Information & Service Center & Number of Service Center \\
\hline & $\begin{array}{c}\text { Dock Operation Status } \\
\text { Port Congestion Information }\end{array}$ & Marine Service Station & $\begin{array}{l}\text { Position of Service Station } \\
\text { Queuing Number of Ships }\end{array}$ \\
\hline & $\begin{array}{c}\text { Estimated Time of Arrival } \\
\text { Traffic Information }\end{array}$ & Refuse Collection Point & $\begin{array}{l}\text { Position of Service Station } \\
\text { Queuing Number of Ships }\end{array}$ \\
\hline \multirow{10}{*}{ Ships } & Position Information & \multirow{7}{*}{ Bridges } & Capacity Information \\
\hline & Speed Information & & Cargos Information \\
\hline & Certification Information & & Estimated Time of Arrival \\
\hline & Payment Information & & Estimated Time of Departure \\
\hline & Illegal Traffic Information & & Traffic Information \\
\hline & Visa Information for Destination & & Short-term Planning of Locks \\
\hline & Loading Information & & Long-term Planning of Ships \\
\hline & Draft Information & & Short-term Planning of Bridges \\
\hline & Owner of Vessels Information & & Long-term Planning of Bridges \\
\hline & Owner of Cargos Information & & Open Time of Bridges \\
\hline
\end{tabular}

\subsection{The Technical Architecture of the Networks Layer}

The networks layer is the fundamental infrastructure of the IoV system and represents the fundamental facilities of the data resources integration and management system. In order to meet the various demands of different businesses and avoid the vulnerability of the IoV link, the networks of $\mathrm{IoV}$ are constructed in various ways.

\subsubsection{Self-assembling Networks of the IoV with Ad Hoc Technology}

An ad hoc network is a kind of wireless networking paradigm for mobile hosts. Originally, the military tactical and other security-sensitive operations were the main applications of ad hoc networks. However, there is a trend of adopting ad hoc networks for civil uses in earthquake and flood relief situations due to their unique properties [18]. Now research into ad hoc technology aims at a routing protocol for adapting the dynamic network topology. The representative achievements are the destination sequenced distance vector (DSDV), the ad hoc on demand distance vector (AODV), dynamic source routing (DSR), the temporally ordered routing algorithm (TORA) and zone routing protocol (ZRP) [19]. The DSDV is a kind of table-driven routing protocol, each node of which needs a routing table containing the routing information to all other nodes [20]. The DSDV takes advantage of the flood searching algorithm to find the route. When the header of the source node carries the destination node address and broadcasts to the adjacent nodes, the intermediate node would judge itself to transfer the message or to receive the message according to the destination node address. The advantage of this method is improving the reliability of transmission, while the disadvantage is that the network costs too much. The DSR is a kind routing algorithm to look up the route based on its need to send data [20]. In the DSR routing protocol, the node does not need to maintain the information. The network could complete self-configuration without any network infrastructure or facilities. The use of DSR routing protocol reduces the maintenance cost of the routing but it could cause competition between different information and prevent the correct communication. In summary, the strengths of 
the DSDV and the DSR are that the AODV is developed to improve the ad hoc network technology [21]. In the AODV routing protocol, there are three control messages including route request (RREQ), route reply (RREP) and the route error (RERR). The node could judge the correction of the message from the native broadcast. If the node finds the link of active routing breaking in the routing table, the node could send the RERR to the host and delete the link in the routing table. The node which received the RERR messages could delete the route including the disconnected link in the routing table. When the route fails to connect to the table due to the source node movement, the source node would make a route request again. The TORA is a kind of distrusted algorithm. It could provide various routing between the source node and destination node [22]. The TORA could distribute a height value to each node and the height value of the destination node is the lowest. As a result, compared with the height value between adjacent nodes, various efficient paths could be established and the direction of the path is from the higher node point to the lower node. The advantage of the TORA is reducing the time delay during the exchange of information.

The application of the ad hoc network technology in the IoV system has the great advantage that using the ad hoc communication to monitor and report the position of the vessels could have a large network latency and does not need the high speed data transmission [23].

However, there are some technical barriers to applying the ad hoc technology in the IoV system. For instance, in the ocean, there is a long distance between ships. From chart data of the vessel traffic services (VTS), it can be seen that ships in ports, wharfs and offshore have a concentrated distribution, while the ships are far from each other in open sea. As a result, it is suitable to use ad hoc network technology in ships distributed in concentrated areas. Otherwise, it is better to choose other networks such as the satellite centric model networks, opportunistic networks and so on.

\subsubsection{Satellite Centric Mode Networks}

The satellite network is one of the best ways to solve the ship communications during navigations. However, the communication bandwidth of the satellite network limits the development of applying satellite communication between the satellites and the ground, as well as the satellites and the ocean. Recently lots of progress has been made to improve the low-bandwidth communication in satellite positioning and satellite communication. Now the United States, Europe, Russia and China have the ability to realize global communications. The network coverage of the International maritime satellite is shown in Figure 6 [24].

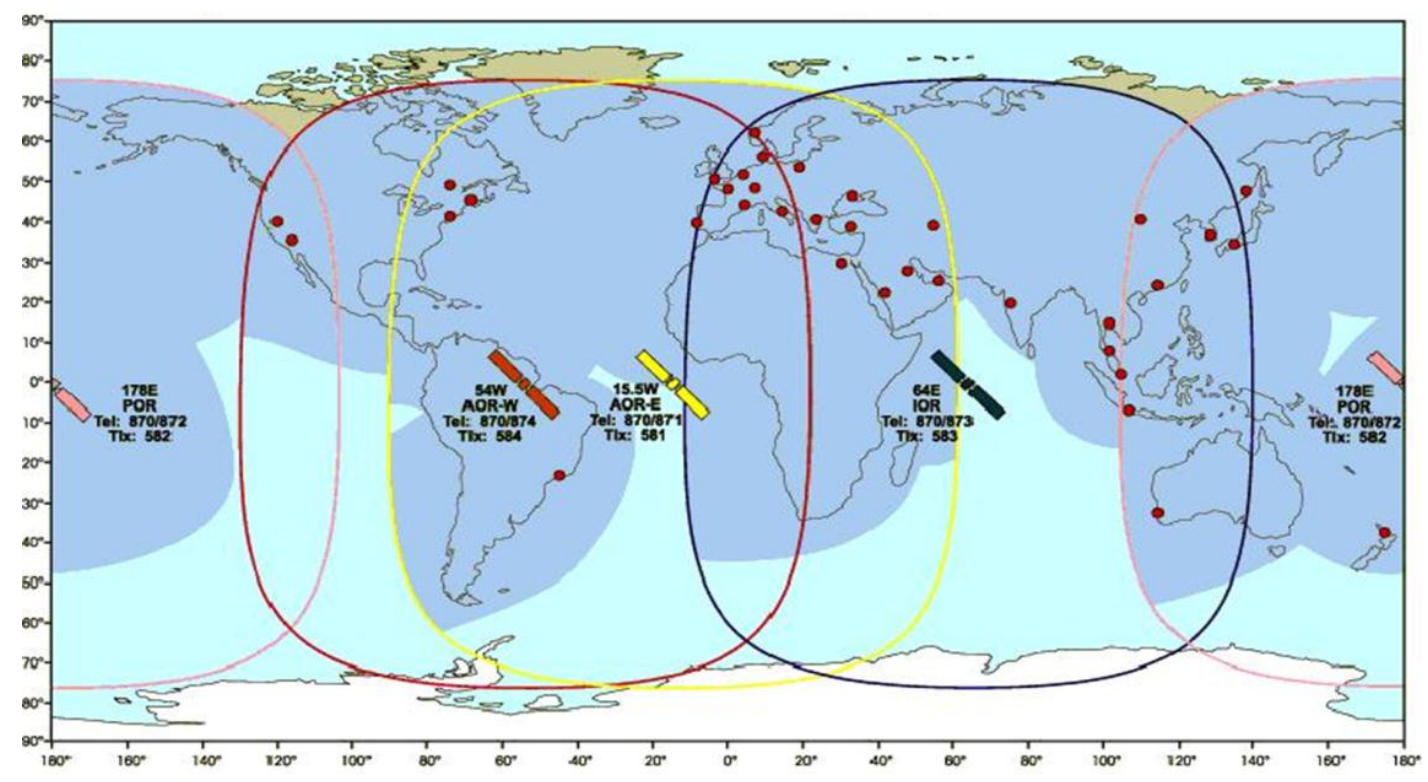

Figure 6. The network coverage of the International maritime satellite. 
At present, the main functions of the global navigation satellite system are navigation and measurement. In future, it can be used in the IoV system. The global positioning system (GPS) of the USA consists of 24 satellites ( 21 of the satellites are working, 3 of the satellites are standby) which are used for military and civil purposes. The Galileo system of the European Space Agency has 30 satellites and is used for civil purposes. Similarly, Russia developed a set of positioning systems, the precision of which is limited to $10 \mathrm{~m}$. The Beidou navigation satellite system from China has 5 geostationary orbit satellites and 30 non-geo satellites [25]. China has carried out lots of studies and practices on the IoV by the Beidou navigation satellite system. Based on the Beidou navigation satellite system (BDS), automatic identification system (AIS), geographic information system (GIS), the internet and mobiles, China is developing a kind of IoV system called Beidou IoV to realize integrated service for the sea, the sky and the ground. The Beidou IoV system covers the eastern coastal areas of China and provides ship monitoring, short message communication and fishing-vessels port management [26].

\subsubsection{Base Station Networks}

The mobile communication network is an important foundation to build the networks of the IoV system which can be used in the coastal base stations, island base stations and river base stations. The mobile communication network which is a nice complement to the marine communication facilities has a variety of access networking technologies such as the general packet radio service (GPRS), wideband code division multiple access (WCDMA), and third and fourth generation telecommunication technologies (3G and 4G) [27]. The GPRS network is developed based on the GSM network [28]. The user datagram protocol (UDP) and transmission control protocol (TCP) are the main communication protocol for the GPRS networks. There are many advantages to using the GPRS technology in the base station networks. The connection cost of GPRS is very cheap and the resource utilization is very high. The GPRS could provide $115 \mathrm{kbps}$ transmission speed. Now a kind network called Wideband Code Division Multiple Access (WCDMA) is widely used in the construction of base station networks [29]. The WCDMA could adapt various transmission rates of the information, ranging from $8 \mathrm{Kbps}$ to $2 \mathrm{Mkbps}$. The development of WCDMA technology realizes global roaming which has great impact on the communication of the IoV system. Currently, the WCDMA technology occupies more than $77 \%$ of the communication market. Apart from the WCDMA technology, 4G uses the orthogonal frequency division multiplexer (OFDM) technology [30]. It could run at the uplink rate of $20 \mathrm{Mbps}$. At the same time, combining with satellite and optical fiber technology, 4G could provide the information services and information collection to realize fast communication between the base station and the ships. Based on the actual needs such as the cost, stability, efficiency and so on, it can choose different technologies to construct the base station networks.

Now a new communication technology, the fifth generation telecommunication technology (5G), is the extension of $4 \mathrm{G}$. Although there are no standards for $5 \mathrm{G}$, the key technologies including the architectures, wireless networking, wireless transmission, radio frequency (RF) and the development of a new spectrum will be quite useful to downscale the base station, remove the traditional nodes and the flat network architecture. With the development of mobile communication technology, the networks of the IoV system will depend on 5G in the future [31].

\subsubsection{Opportunistic Networks}

The opportunistic network is a kind of network which takes advantage of the encounter brought by the movement of nodes to realize the network communication [32]. It is not necessary to establish the full path between the source node and destination node [33]. The ad hoc networks and wireless networks always lack treatment plans to deal with network interruption in difficult environments [34]. As a result, when the network is interrupted, the network performance may decrease dramatically. Furthermore, in practice, the topological structure of the adaptive wireless network may change at any time, which cannot guarantee the connection between the source node and destination node. Against this background, the opportunistic network technology has been developed. 
Now various sensors are arranged in the ocean to collect the ocean data. It can use the storing-carrying-transferring mechanism of opportunistic networks to store information in the airplanes and ships passing through these areas and transfer the information data to other places when the airplanes and ships arrive to the land [35].

\subsubsection{Underwater Acoustic Communication Networks}

As well as the land communication, the underwater communication can be divided into wire communication and wireless communication. Laying electric and optical fiber submarine cables realizes the underwater wire communication, while the underwater wireless communication networks can be regarded as delay tolerant networks (DTNs) due to their sparse deployment, node mobility and some severely impaired bandwidth-limited underwater acoustic communication channels [36]. As a reference implementation of the DTNs protocol, DTN2 was designed and implemented [31]. Based on the DTN2 research platform, an underwater convergence layer is developed to interconnect acoustic networks [37]. The most advanced underwater acoustic communication equipment is now applied in military, special commercial and scientific research [38]. The distance of signal transmission can be several thousand meters.

\subsection{The Technical Architecture of the Data Resource Layer}

The data resource layer is the core layer of the IoV system. Therefore, in this layer, it is very important to take the information resources in scientific classification with the standard of the unified data exchange to ensure the reliability of the data. Figure 7 is the architecture of the data resource layer.

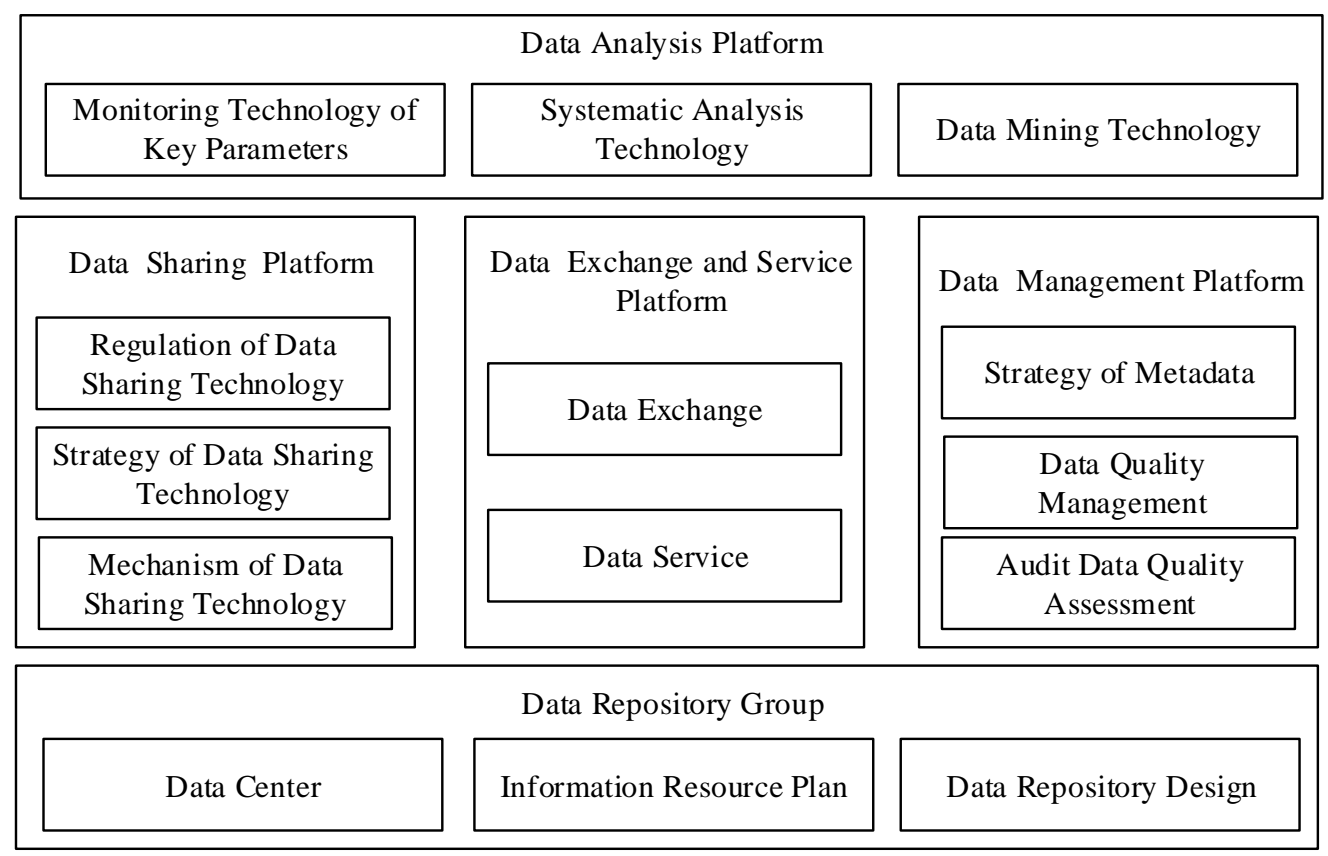

Figure 7. The technical architecture of the Data Resource Layer.

As can be seen in Figure 7, there are several databases, such as the business databases, the basic databases and the theme database groups. With these databases, a variety of theme databases would be developed by classifying the sensor information and the requirements of administration, customers and crews. At the same time, the architecture of the data resource layer needs to obey the standard regulations, including the uniform information coding, uniform database naming and uniform management [39]. 
In order to provide a large amount of data to the upper layer, the data resource should support the present mainstream databases such as the MySQL, SQL server, Oracle, Text, Excel, XML and so on [40]. At the same time, the data resource layer should also have the data interfaces with different facilities. According to the data conversion components, the data resource could solve various complicated problems by the permutation and combination. Now the big data processing platform has realized the massive data storage and mining. The speed of data update could be achieved in milliseconds. With these advanced data resource technologies, the IoV system could store huge amounts of data from different ships, ports and the base stations by the perception facilities.

\subsection{The Technical Architecture of the Application Layer}

The main function of the application layer is providing service for the administration, enterprises, crews, ship owners and so on. The customers select the service from the client service list and then the service request schedules the corresponding resources through the management system [41]. Lastly, the web applications are deployed.

For example, based on the Yangtze River Delta inland water transportation public information service platform, Dai [41] established a kind of application layer which is shown in Figure 8. It can be seen that in the application layer is a core layer in the IoV system. With the data resource layer's support, variety of the application systems is developed in this layer. In order to meet the requirement of the business management and public information service, the functions including the shipping company and crew management, traffic assessment information, meteorological information, policies services, logistics information, dangerous goods declaration and channel congestion prediction are developed and integrated into several multi-platforms or systems. Based on the data analysis results, each platform could help the administrators make a good decision.

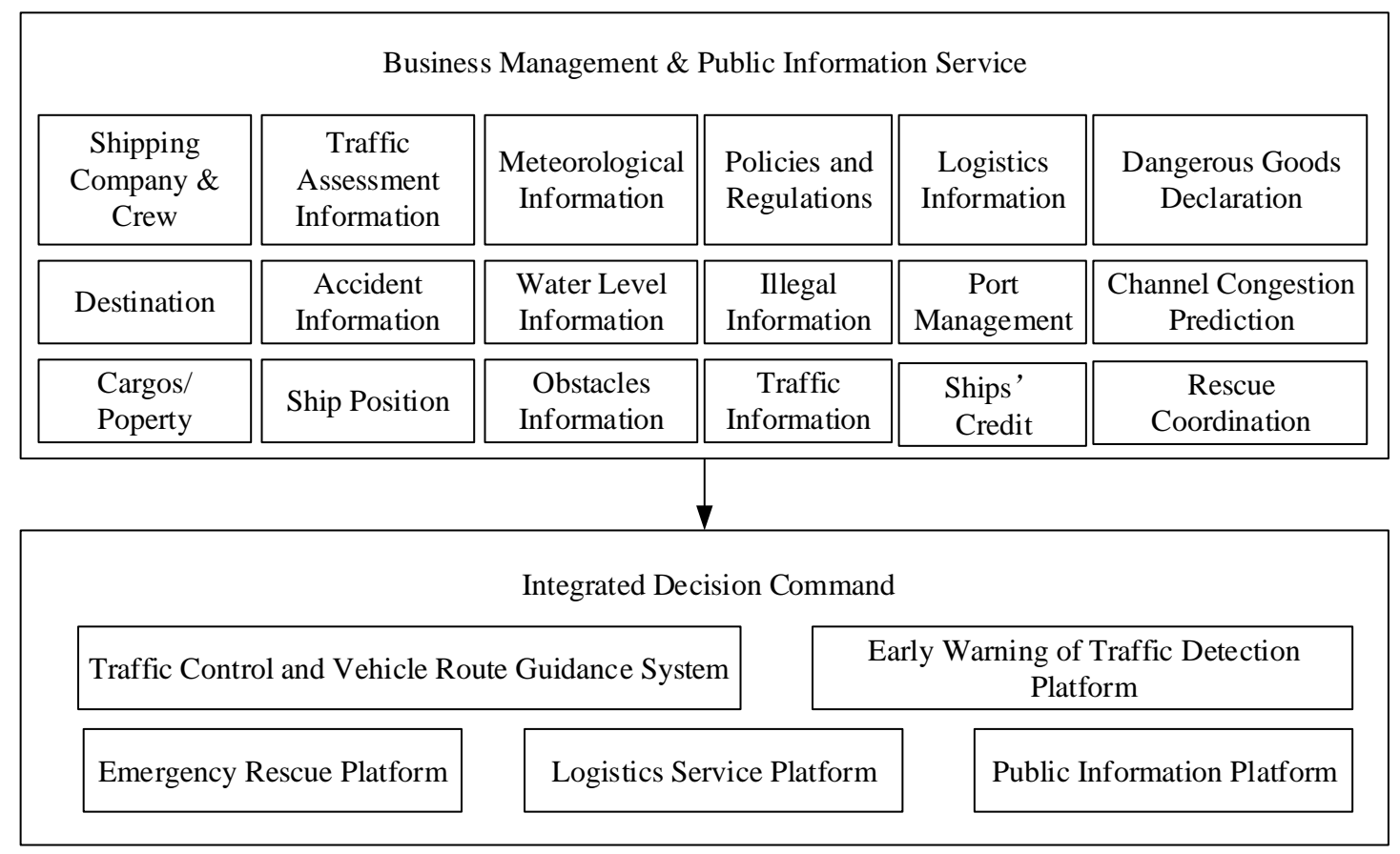

Figure 8. The technical architecture of the Application Layer.

\subsection{The Technical Architecture of the Exhibition Layer}

The exhibition layer is a platform to publish the service information to the public in real time, which is a useful supplement for personalized service and business management [42,43]. The exhibition layer can communicate with various devices and platforms, as shown in Figure 9. 


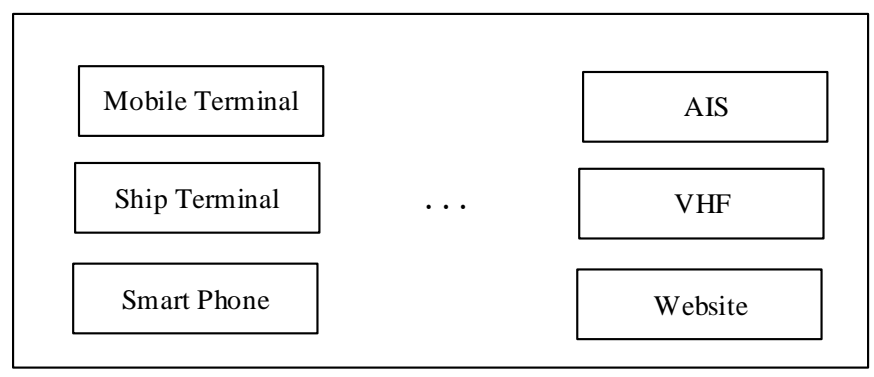

Figure 9. The architecture of the Exhibition Layer.

\section{Characteristics of the Internet of Vessels}

\subsection{Information Processing and Information Fusion}

In the IoV system, the objects of the information collection are bridges, ships, ship locks, channels, meteorology and hydrology and so on. All the information is in static and dynamic states. Due to the variety of the information sources, the data formats of different sources are quite different. The information from the IoV system has the feature of multi-source heterogeneous [44-46]. Therefore, the information fusion becomes the most important stage of the intelligent information processing. The information fusion is a kind of multi-level, multi-aspect information process including multi-source data detection, correlation, combination and estimation. It could obtain the results and the corollaries with more accuracy than that from the single sensor. When one part or several parts of the system cannot operate normally, the information fusion technology could keep the system working well according to the information data collected from the other parts of the system. Due to the overlap and synergy among a variety of sensors, the information fusion could obtain as much data in the time and space domain, which means that the sampler info will be covered more widely and the object will be described more comprehensively. In order to ensure the credibility of the information, the information fusion technology describes the same object in multiple dimensions. The information fusion technology does not require much to the sensors. As a result, the sensors could be designed and installed in a flexible way.

The distributing structure of the information fusion technology preprocesses the sounding data to generate tracking information and then make a global judgement in the center [47]. The structure of the information fusion is shown in Figure 10.

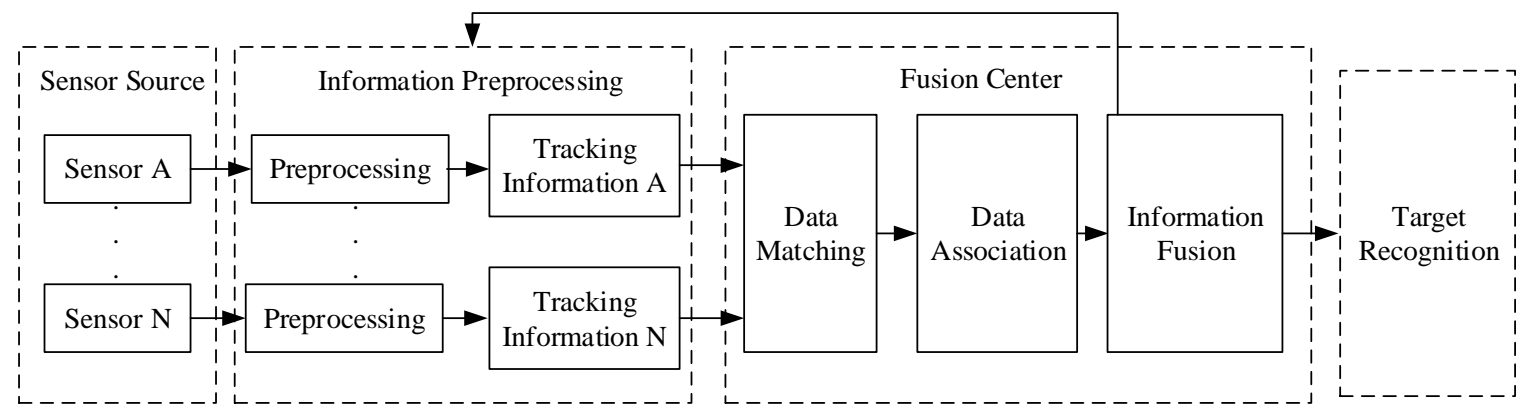

Figure 10. The distributing structure of the information fusion technology.

$\mathrm{Yu}$ [48] describes a detailed case of the information fusion between the VTS (Vessels Traffic System) and the AIS (Automatic Identification System) to indicate the importance of the characteristics in the IoV system. As parts of the IoV system, the VTS system and AIS system have their own functions and facilities. In the VTS system, the radar is the main facility to collect the information and data. Therefore, most characteristics of the VTS system are similar to that of the radar. Generally, the VTS 
system has accuracy in monitoring and tracking the ship moving status information, while it has great error in the location determination. In addition, the VTS system has a good extension to fuse a lot of of heterogeneous information. The AIS system is a network system to exchange and share the navigational information between different ships through the VHF link. Based on the maritime mobile service identity, the AIS system could track various ships separately and get their inherent information including the type, the size and the tonnage accurately. The AIS system also has the ability to get the ships' dynamic information including the location, speeds and navigational status information. As a result, the information fusion technology could fuse the advantages of these two different systems in the fusion center. The AIS system could help improve the location information accuracy of the VTS system, while the VTS system makes full use of its own advantages on capturing and tracking the dynamic information. On the other hand, the AIS system should be mounted on the ships, so it has a high requirement on the equipment. The VTS system just uses the radar to gain the information of ships, which does not rely on the terminals. With the help of the information fusion technology, the IoV system will be efficient and well managed. The Kalman algorithm method [49] is used in the information fusion of VTS and AIS. Following the process in Figure 10, the information collected from sensors is processed by the Kalman algorithm to get the trajectory, then the information from the AIS system and VTS system is gathered and classified in the fusion center. In the fusion center, the temporal and special alignment technology and the track correlation technology solve the problems of the unity of the time domain and spatial domain between the AIS system and VTS system. Finally, with the fusion of related trajectory data, the administration could get more stable and more precise ship position information and movement information.

Meanwhile, the information fusion technology could help the IoV system choose the correct strategy and prediction. Borkowski [50] presented an algorithm of ship movement trajectory prediction through data fusion which considers the measurements of the ship's current position from different kinds of information sources such as the ARPA (automatic radar plotting aid), GNSS (global navigational satellite system), AIS (automatic identification system) and ENC (electronic navigational chart). Based on the information fusion, Borkowski verified the algorithm to improve the reliability and accuracy of prediction. Currently, the navigation data fusion has been implemented in navigational decision support systems and used on board many ships.

Similarly, there are many other sub-systems of the IoV system using the information fusion technology such as the fusion between the Radio Frequency Identification (RFID) system and the Zigbee wireless networking system [51]. The main goal of the IoV system using the information fusion technology is to improve efficiency and accuracy.

\subsection{Data Analysis Methods}

Depending on the VTS, AIS, GIS, CCTV and RFID, the maritime administration obtains the dynamic data of the ships. With the complete ships databases, crew databases, meteorological databases and hydrologic database, it establishes a big data platform. Traditional data analysis methods are sampling or filtering the raw data and analyzing the data sample to look for patterns and features. Agnieszka [52] proposed an approach of ant colony optimization (ACO) to path the ship planning to avoid collision. In the research, the ACO-based algorithm was constructed including the data initialization, solution construction, pheromone trail update and the objective function. According to the scenarios shown in Agnieszka's research, the algorithm could help ships hedge their collision risks and satisfy rule 15 of COLREGs (Convention on the International Regulations for Preventing Collisions at Sea). Therefore, it can be seen that the most important characteristic of the traditional data analysis method is the complicated algorithm to get as much information as possible. Apart from the traditional data analysis method, the big data analysis method of the IoV system could analyze the whole databases efficiently [53].

At present, there are several methods of big analysis used for the IoV system, such as the deep learning approach, the knowledge calculation method and the visualization method. 
(1) The deep learning approach usually uses the artificial neural network method. Based on the multi-layered framework, this method could analyze and express the data. Taking advantage of data volume from the big data platform, it can use a more complicated model to represent the data effectively. Now this method is widely applied in the prediction of ship operation [54], collision warning [55] and risk analysis [56]. Zhao [54] took the turning performance of a single screw ship as an example to establish a mathematical model based on the artificial neural network. As was known, when the ship conditions (loading, oil consumption, speed, trim angles and heel angles) and the environment (wind, waves, water flow, offshore voyage) changed, the characteristics of ship operation would change at the same time. Based on the big data from the IoV system, the artificial neural network was trained to meet the predicted requirement for the ship's operation. According to the experiment with the real ship, the reliability of this method was verified. Lu [55] established a kind of collision risk evaluating model by the neural network. Due to the big data analysis, the collision risk model obtained good results and could provide a reference for increasing the safety of the ship.

(2) The knowledge calculation method extracts valuable knowledge from the big data platform to construct searchable and computable knowledge bases that include knowledge base construction, integration of multiple knowledge and knowledge update. The integration of multi-knowledge considers the sharing and reusing of knowledge and improves the real time and effectiveness of the data. This method is widely applied in the VTS [57], ship navigation [58] and decisions regarding energy consumption for ships [59]. Zbigniew [60] developed a kind of information system supporting navigational decision making. Information was acquired in the information acquisition mode and then the situation analysis would calculate and process the data. With the results of the knowledge calculation, the system made a good choice and took action to help the ship avoid collision. Mladineo [61] studied a multi-criteria analysis-based decision support system developed for the management of incidents in maritime traffic. The decision support system organized a variety of information data related to emergency management-spatial data, radar data, weather data and GIS data-for the administrator, in a comprehensible and user-friendly way. It used the preference ranking organization method for enrichment evaluations (PROMETHEE) for treating the multi-criteria problem. A case on the east coast of the Adriatic Sea was studied to indicate that this method was more understandable and effective.

(3) The visualization method, according to the interactive information display and high dimensional dynamic information, can make a decision in real time. Taking advantage of the visualization method, the ships' traffic flow model is built to master the state of the ship traffic flow, to display the degree of channel crowding in a visualization, to assess the security risks and to give early warning of the security information [62]. Based on the big data, Robin [54] described the advantage of the description and illustration being used to present the information. With the description and illustration, the decision maker could be provided with the most essential and salient aspect of a given analysis quickly. Similarly, when this method was used in the IoV system, the administrators would judge the case in a direct way, and make a quick response for alarm and emergency. Meanwhile, it was also a good way to alleviate the traffic in the inland rivers or in the ports.

\section{Applications and Benefits of the IoV}

\subsection{Intelligent Navigation of Ships}

The IoV system makes it possible to realize the intelligent navigation of ships. The IoV system has a variety of functions including the completed navigation, collision avoidance, information display, monitor, alarm, satellite communication, ship management and so on, which are convenient for the crew and shore-based commander to observe, operate and optimize the information process collected 
from the equipment. The use of the IoV system will play a greater role in the guarantee of ship safety navigation than before [63].

With the perception information including the ship navigating state, environmental factors and the ship operating data obtained from the sensors, the IoV system will take advantage of the data resource layer to distribute and share these data and choose the optimal path to operate the ship automatically.

With the development of unmanned control theory, the driverless car and unmanned aerial vehicle have become reality. The IoV system is quite helpful for research on unmanned ships. In the inland river, due to the complicated channel environment and the traffic flow density, the unmanned ships have high requests for obstacle perception, route planning and tracking ability. As described in Sections 2 and 3, the IoV system has the ability to meet these requirements for the unmanned ships. Figure 11 is the schematic diagram for the ship automatic collision avoidance [64].

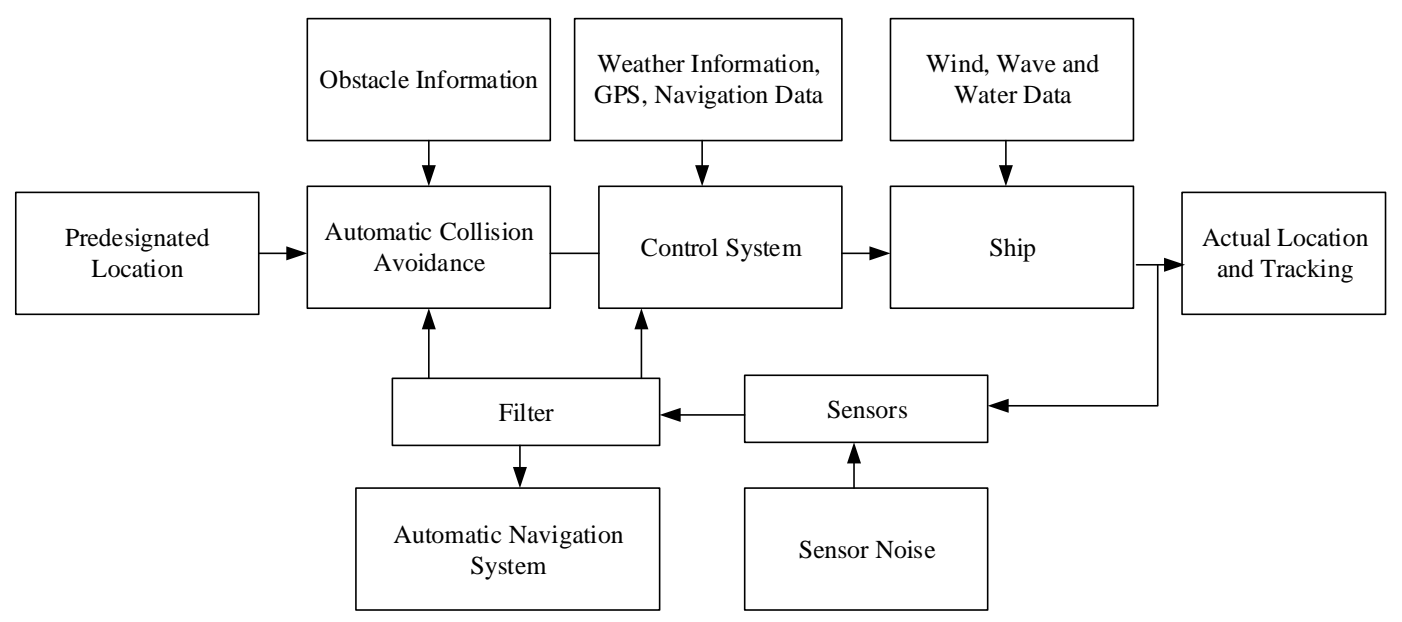

Figure 11. The principle of the ship automatic collision avoidance.

\subsection{Intelligent Management and Service of Vessels}

With the help of the IoV system, managers could get the accurate and complete real-time movement of the ships and predict the potential traffic problems. As a result, they can make reasonable plans and project emergencies to prevent disasters and reduce property loss. Based on the VTS, AIS and GIS, Kao [65] proposed a fuzzy logic method to predict vessel collision risk to improve the safety of navigation and the efficiency of the ship management. Wen [66] took advantage of artificial neural network and logistic regression to distinguish the smuggling ships. Based on the classification of different ships, by following their types, drafts and destinations, Wijaya [67] analyzed the routes of thousands ships to realize the predicted behavior for the target ship in crowded water areas.

The intelligent service of vessels from the IoV system allows service providers to identify the customers' (ship owners, ship crews) explicit and implicit demands, and then meet their requirements efficiently [68]. For instance, according to the information from the IoV, it can provide personalized management (Visa, cargo loading and food configuration, sailing schedule setting), set the optimal route for ships, develop monitoring and adjust the plan of energy consumption for the ships. With funding from the European Union, the European institutions are accelerating the construction of IoV systems to realize electronic freight, which is shown in Figure 12 [69]. 


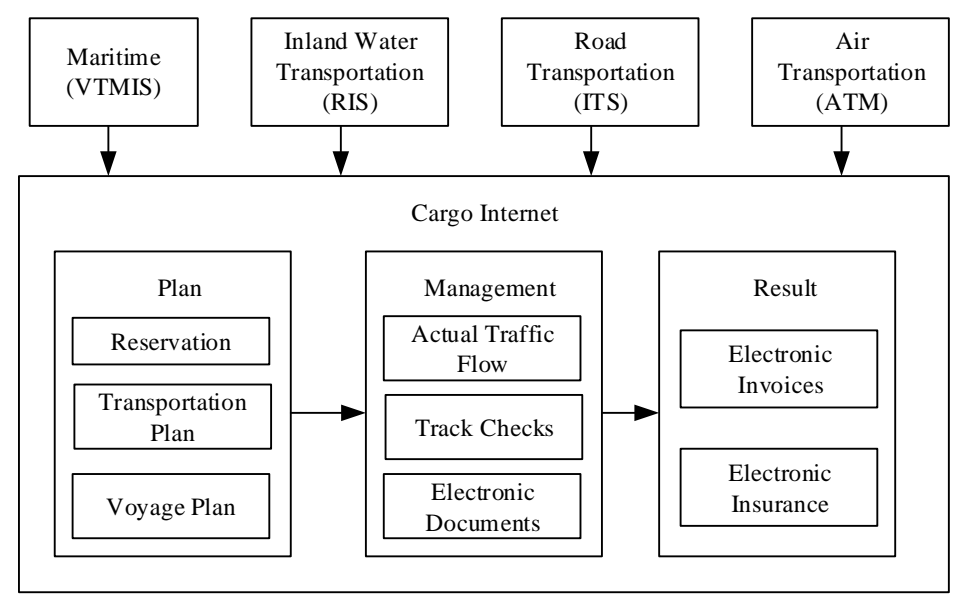

Figure 12. The schematic diagram of the electronic freight.

\subsection{Traffic Flow Prediction}

One typical application of $\mathrm{IoV}$ is for traffic flow prediction. The theory of traffic flow is the basic theory of the intelligent ship transportation management. With the help of the IoV system, it can analyze the dynamic laws of the ships and the changes in the relationship between the traffic speed and the traffic density. Taking advantage of the prediction model and the information data obtained from the IoV system, the traffic flow prediction could predict the short-term, medium-term and long-term traffic. With the development of the IoV system, a number of advanced models are proposed to predict the traffic flow. Li [70] used an improved model of robust support vector regression to predict the traffic flow of Tianjin Port. Based on the port's characteristics, ship behavior and historic data of the traffic flow, Liu [71] established a novel integrated traffic flow prediction model. Due to the problems of ship navigation safety and congestion caused by the increasing ship traffic and ship size in Pohang Port, Song simulates the ships' tracks in real time and the traffic flow to provide the best route guidance for ships. Xu [72] adopted the Monte-Carlo method to analyze the history data of traffic flow in the Wuhan bridge area of Yangtze River and to propose a generative approach to improve the safety of ship navigation. It can be seen from this, if these traffic flow prediction models are added to the IoV data resource layer and give the appropriate results in the application layer, they are quite useful for improving the safety of the ship navigation and the utilization of the ports, to promote the development of the intelligent management.

\section{Challenges and Prospects}

\subsection{Challenges of the IoV System}

\subsubsection{Safety Mechanism}

Compared with the traditional networks, due to the changes of ship trajectory and the unstable topology structure, the network of IoV is a dynamic, temporary network [73]. In the effective range of communication, the nodes of IoV networks keep a connection. When the distance of nodes goes out of scope, the connection will break immediately [74]. In addition, because of the ad hoc technology among the vessels, the wireless networking structure between the shore and ships as well as the wired network structure between the data sensing equipment and the databases, the networks of IoV are in multi-network integration conditions. Therefore, the IoV system has more security issues than the traditional networks.

The IoV has the features of centerless and self-organization so it cannot use the safety mechanism of traditional networks directly [75]. Meanwhile the sensing nodes of the IoV system are deployed in an environment that needs nobody to see or control. As a result, the IoV will be under threat 
from information leakage, information tampering, congestion attack and flooding attack. The attacker could obtain the information data from the sensing nodes and break into the networks of the IoV system. The other challenges of the IoV system with reagards to the safety mechanism are ensuring the authentication technology based on the user identity security, the establishment of security keys' transmissions, the security of data encryption and the users' privacy protection [76].

\subsubsection{The Limit of Transmission for the Waterway Traffic Information}

Although the waterway traffic has many ways, such as perception and communication, to transfer information from vessel to vessel, vessel to shore, vessel to buoy, limits of transmission still exist in use, arrangement and integration [77]. The details of the limitation are described below.

(1) As is well known, there are a variety of communication styles such as VHF, RFID, GPRS and $3 G[78,79]$. The data of these communication styles is similar but not compatible because of the lack of unified interaction interfaces.

(2) Due to the lack of service contents $[80,81]$ and service styles, the information is transferred from the ship terminal to the coast station only to meet the requirement of the administration. A general service platform should be established to provide various information services.

(3) When the ships sail in different regions, the information will be transferred across these regions. If the business systems in these regions do not support each other, it is a barrier for the information sharing and information efficiency.

\subsection{Prospects of the IoV System}

With the development of the IoV, it will study more advanced ship intelligent terminals and buoy intelligent terminals to enrich the means of perception. The integration of these communication technologies such as 3G, 4G, AIS and RFID will realize intelligent switching between one another. The data information collected from the sensors will be more compatible. The integration information platform of the IoV system with the ship-shore interaction will be more comprehensive and advanced.

In future, a variety of novel heterogeneous networks will be applied in the IoV system. Meanwhile, the IoV system will realize the networks switching seamlessly to increase efficiency and reduce the operating costs.

\section{Conclusions}

In this paper, the IoV is introduced. With the development of big data, the Internet of Things and cyber-physical systems, the IoV will be a burgeoning research area in the ship industry. The publications mentioned above provide strong support for the building of the IoV system. Its architecture, characteristics and relevant application have been described. The construction of the IoV system is still in the primary stage and it is always accompanied by various challenges. Technical innovation and ambitions of overcoming obstacles are the driving force promoting the technical advancement and development of the IoV system. The future research priorities can take, but are not limited to, the following several directions:

(1) Exchange ability of data fusion of heterogeneous networks in water transport,

(2) Intelligent ship management and service techniques,

(3) Intelligent control techniques for real-time practical usage,

(4) Cross-regional information integration and resource management in IoV,

(5) Information security protection for large scale ship network communication systems,

(6) The application of new generation wireless communication technology.

In the future, the IoV system will play an important role in shipping transportation all over the world. Many advanced technologies will be developed for IoV. The authors are currently undertaking state-of-the-art research into the automatic identification system (AIS) for ship identification in 
large-scale ship networks, and big data learning for real-time monitoring and management of ships. The outcomes will be reported in due course.

Acknowledgments: This research is sponsored by the National Natural Sciences Foundation of China (NSFC) (51709244, 51522906, 51479184, 51606178), the Excellent Youth Foundation of Shandong Scientific Committee (JQ201512), Yingcai Project of CUMT (YC20170001) and the Taishan Scholars Program of Shandong Province.

Author Contributions: Fushun Liu and Zhe Tian designed the idea; Fushun Liu and Yingchun Xie provided the materials; Zhe Tian, Fushun Liu, Zhixiong Li and Reza Malekian wrote the manuscript.

Conflicts of Interest: The authors declare no conflict of interest.

\section{References}

1. Qin, W.M.; Wang, X.F. A survey on netting with Internet of Vessels. Navig. China 2015, 38, 1-4.

2. Liu, C.G.; Chu, X.M.; Xie, S.; Yan, X.P. Review and prospect of ship intelligent. Ship Eng. 2016, 38, 77-84.

3. Preece, A.; Decker, S. Intelligent web services. IEEE Intell. Syst. 2002, 17, 15-17. [CrossRef]

4. Oberheim, C.; Heinz, M.; Steinhuber, L.; Blesenkemper, J. Status of RIS in Germany part 1: New information systems for inland navigation in Germany. In Proceedings of the 30th PIANC-AIPCN Congress 2002, Sydney, Australia, 22-26 September 2002; pp. 1957-1966.

5. Vallant, J.; Hofmann-Wellenhof, B. River information services. Elektrotechnik Informationstechnik 2008, 125, 239-243. [CrossRef]

6. Zhou, J.H.; Zhang, F.J.; Gong, Y.P.; Zhang, Y. Data exchange research and thinking of the Europe inland river shipping integrated information service system. China Water Trans. 2011, 11, 83-85.

7. Racetin, I. Croatian river information services. In Proceedings of the International Cartographic Conference, Santiago, Chile, 15-21 November 2009.

8. Svetlana, M.; Milan, M.; Ivan, S. European policy for the promotion of inland waterway transport: A case study of the Danube River. Afr. J. Bus. Manag. 2012, 6, 2498-2507.

9. Luo, B.C.; Xie, Y.L. An overview of the Europe inland river shipping integrated information service system. Ship Manag. 2007, 29, 37-39.

10. European Communities. White Paper European Transport Policy for 2010: Time to Decide; Office for Official Publications of the European Communities: Luxembourg, 2001; ISBN 9789289403412.

11. Kazimierski, W.; Wawrzyniak, N. Exchange of navigational information between VTS and RIS for inland shipping usere needs. In Proceedings of the 14th International Conference on Transport Systems Telematics, Katowice, Poland, 22-25 October 2014.

12. Dong, Y.H.; Sun, W.; Dong, L.H.; Zhang, C.L. On construction of Internet of Ships. Prot. Waterw. Eng. 2012, $469,145-149$.

13. Dai, M. The Key Technologies Research of Vessels Connected to Internet Information Perception and Interaction in Yangtze River Delta. Ph.D. Thesis, Chang'an University, Xi'an, China, 2016.

14. Wang, T.C.; Xie, Y.Z.; Yan, H. Research of multi sensor information fusion technology based ono extension neural network. Math. Model. Eng. Probl. 2016, 3, 129-134. [CrossRef]

15. Yang, L. Research on fusion technology of fuzzy system and neural network. In Proceedings of the 5th International Conference on Machinery, Materials and Computing Technology (ICMMCT 2017), Beijing, China, 25-26 March 2017. [CrossRef]

16. Xiang, M.; Wang, T.T.; Luo, Z.Y.; Cai, L.Q.; Huang, J.J.; Hou, X.Z. Design of test platform for electricity information collection system based on multi-network fusion technology. In Proceedings of the 2013 IEEE International Conference of IEEE Region 10 (TENCON 2013), Xi'an, China, 22-25 October 2013. [CrossRef]

17. Bai, K. Reseasrch on Internet of Ships RFID Data Process. Ph.D. Thesis, Dalian Maritime University, Dalian, China, 2013.

18. Zhou, L.D.; Haas, Z.J. Securing ad hoc networks. IEEE Netw. 1999, 13, 24-30. [CrossRef]

19. Arun Kunmar, B.R.; Reddy, L.C.; Hiremath, P.S. Performance comparison of wireless mobile ad-hoc network routing protocols. Int. J. Comput. Sci. Netw. Secur. 2008, 8, 337-343.

20. Huang, Y. A Terminal Display System of Maritime Communication Based on Ad Hoc Network. Master's Thesis, Dalian Maritime University, Dalian, China, 2010. 
21. Zhou, J.Y. The application on ad hoc network in ship communication terminal system. Ship Sci. Technol. 2016, 38, 103-105.

22. Chen, H.Y. Investigation on TORA Routing Protocol in Ad Hoc Networks and Its Improvement. Master's Thesis, Hubei University, Wuhan, China, 2013.

23. Frank, K.; Stefan, S.; Andreas, K.; Alfred, G.; Michael, W. Securing ad hoc routing protocols. In Proceedings of the EUROMICRO Conference, Rennes, France, 31 August-3 September 2004; p. 519.

24. Joytom. Available online: http:/ / www.joytom.cpooo.com/news/17201.html (accessed on 6 July 2009).

25. Yang, Y.X. Progress, contribution and challenges of Compass/Beidou Satellite Navigation System. Acta Geod. Cartogr. Sin. 2010, 39, 1-6.

26. Hu, G.; Ma, X.; Fan, Q.Y. The applications of Compass Navigation Satellite System to marine fishing industry. Fish. Mod. 2010, 37, 60-62.

27. Osseiran, A.; Boccardi, F.; Braaun, V. Scenarios for 5G mobile and wireless communications: The vision of the METIS project. IEEE Comput. Mag. 2014, 52, 26-35. [CrossRef]

28. Wu, J.; Chen, Y.B.; Zhong, B.; Zhou, G. Design of ship's data transmission device based on GPRS network. Mar. Electron. Eng. 2015, 35, 55-59.

29. $\mathrm{Xu}, \mathrm{Y} . \mathrm{M}$. The Research and Design of Remote Monitoring System of a Boat Equipment Data Based on 3G Technology. Master's Thesis, Dalian Maritime University, Dalian, China, 2013.

30. Tang, X.Y. Application of 4G communication technology in ship navigation information system. Ship Sci. Technol. 2014, 36, 148-151.

31. Waleed, E.; Alagan, A.; Muhammad, A.L. Internet of Things (IoT) in 5G wireless communications. IEEE Access 2016, 4, 10310-10314. [CrossRef]

32. Pelusi, L.; Passarella, A.; Conti, M. Opportunistic networking: Data forwarding in disconnected mobile ad hoc networks. IEEE Comput. Mag. 2006, 44, 131-141. [CrossRef]

33. Liu, C.H.; Xue, F. Network coding for two-way relaying: Rate region, sum rate and opportunistic scheduling. In Proceedings of the IEEE International Conference on Communications, Beijing, China, 19-23 May 2008.

34. Zhang, Z.; Greiner, A.; Taktak, S. A reconfigurable routing algorithm for a fault-tolerant 2D-Mesh Network-on-Chip. In Proceedings of the IEEE 45th ACM/IEEE Design Automation Conference, Anaheim, CA, USA, 8-13 June 2008.

35. Chen, W.; Letaief, K.B.; Cao, Z. Opportunistic network coding for wireless networks. In Proceedings of the IEEE International Conference on Communications, Glasgow, UK, 24-28 June 2007.

36. Fall, K.; Farrell, S. DTN: An architectural retrospective. IEEE J. Sel. Areas Commun. 2008, 26, 828-836. [CrossRef]

37. Scott, K.; Burleigh, S. Bundle Protocol Specification. Available online: http://tools.ietf.org/html/rfc5050S (accessed on 21 May 2016).

38. Meranim, D.; Berni, A.; Potter, J.; Martins, R. An underwater convergence layer for disruption tolerant networking. In Proceedings of the Baltic Conference on Future Internet Communications (BCFIC 2011), Riga, Latvia, 16-18 February 2011.

39. Berger, C.R.; Zhou, S.; Preisig, J.C. Sparse channel estimation for multicarrier underwater acoustic communication: From subspace methods to compressed sensing. IEEE Trans. Signal Process. 2009, 58, 1708-1721. [CrossRef]

40. Hu, Y.X.; Ma, X.N.; Zheng, L.J. Research of data management in IOT. Internet Things Technol. 2014, 4, 79-82.

41. Dai, M.; Duan, J.; Qian, S. Research on the overall technical framework of the ship networking system in the Yangtze River Delta. In Proceedings of the 2017 2nd International Conference on Information Technology and Management Engineering (ITME 2017), Beijing, China, 15-16 January 2017.

42. Stietencron, M.V.; Rostad, C.C.; Henriksen, B.; Thoben, K.D. Utilising the Internet of Things for the management of through-life engineering services on marine auxiliaries. In Proceedings of the 5th International Conference on Through-Life Engineering Services, Cranfield, UK, 1-2 November 2016.

43. Gubbi, J.; Buyya, R.; Marusic, S. Internet of Things (IoT): A vision, architectural elements, and future directions. Future Gener. Comput. Syst. 2013, 29, 1645-1660. [CrossRef]

44. Guo, M.; Wei, F. Research on key technologies of information fusion based on the marine network. Ship Sci. Technol. 2016, 38, 103-105.

45. Wang, F.; Hu, L.; Zhou, J. A semantics-based approach to multi-source heterogeneous information fusion in the internet of things. Soft Comput. 2017, 21, 2005-2013. [CrossRef] 
46. Yang, X.B.; Zhang, W.Q.; Wu, X.R.; Zhu, L.; Zheng, X. A heterogeneous ship formation network selection algorithm based on service level and load balance. In Proceedings of the 2015 International Conference on Communications, Signal Processing, and Systems, Chengdu, China, 23-24 October 2015.

47. Lv, Z. Multi-Source Information Fusion Technology and Its Application. Master's Thesis, North China Electric Power University, Beijing, China, 2011.

48. Yu, J. Research on the Key Technology of VTS and AIS Information Fusion in the Internet of Inland Ships. Master's Thesis, Wuhan University of Technology, Wuhan, China, 2013.

49. Wang, T.T. Research on Fusion Algorithm of AIS and Radar Dynamic Data. Master's Thesis, Dalian Maritime University, Dalian, China, 2012.

50. Borkowski, P. The ship movement trajectory prediction algorithm using navigational data fusion. Sensors 2017, 17, 1432. [CrossRef] [PubMed]

51. Zhuang, Y.; Song, S.Q. Use of Internet of Things for ship management of inland rivers. In Proceedings of the 2013 Second International Conference on Transportation Information and Safety, Wuhan, China, 29 June-2 July 2013.

52. Lazarowska, A. Ship's trajectory planning for collision avoidance at sea based on ant colony optimisation. J. Navig. 2015, 68, 291-307. [CrossRef]

53. Zhang, Y.; Chen, M.; Liao, X.F. Big data applications: A survey. J. Comput. Res. Dev. 2013, 50, $216-233$.

54. Zhao, X.J.; Xiong, W.H. Ship maneuverability prediction based on modified BP neural network. J. SSSRI 2006, 29, 48-51.

55. Lu, Z.Y. Gnetic neural network algorithm in the application of determining collision risk. Ship Sci. Technol. 2016, 38, 85-87.

56. Alyami, H.; Yang, Z.L.; Riahi, R.; Bonsall, S.; Wang, J. Advanced uncertainty modelling for container port risk analysis. Accid. Anal. Prev. 2016. [CrossRef] [PubMed]

57. Sun, Y.D. The Application of Multi-Source Information Fusion Technology in VTS. Master's Thesis, Dalian Maritime University, Dalian, China, 2014.

58. Wang, P. The Technology Research of Ship Integrated Navigation Multi-Source Information Fusion. Master's Thesis, Jiangsu University of Science and Technology, Zhenjiang, China, 2014.

59. Xia, R.F.; Wan, L.J. Decision support system of ship energy consumption based on knowledge fusion. Ship Eng. 2014, 36, 166-169.

60. Zbigniew, P.; Piotr, W.; Piotr, B. Decision support in collision situations at sea. J. Navig. 2016, 70, 447-464.

61. Mladineo, N.; Mladineo, M.; Knezic, S. Web MCA-based decision support system for incident situations in maritime traffic: Case study of Adriatic Sea. J. Navig. 2017, 70, 1-23. [CrossRef]

62. Hogarth, R.M.; Soyer, E. Using simulated experience to make sense of big data. MIT Sloan Manag. Rev. 2015, $56,49-54$.

63. Kawaguchi, A.; Inaishi, M.; Kondo, H.; Kondo, M. Towards the development of intelligent navigation support systems for group shipping and global marine traffic control. IET Intell. Transp. Syst. 2009, 3, 257-267. [CrossRef]

64. Perera, L.P.; Carvalho, J.P.; Guedes Soares, C. Autonomous guidance and navigation based on the COLREGs rules and regulations of collision avoidance. In Proceedings of the Advanced Ship Design for Pollution Prevention, Split, Croatia, 23-24 November 2009; pp. 205-216.

65. Kao, S.L.; Lee, K.T.; Chang, K.Y. A fuzzy logic method for collision avoidance in Vessel Traffic Service. J. Navig. 2007, 60, 17-31. [CrossRef]

66. Wen, C.H.; Hsu, P.Y.; Wang, C.Y.; Wu, T.L. Identifying Smuggling Vessels with Artificial Neural Network and Logistics Regression in Criminal Intelligence Using Vessels Smuggling Case Data; Springer: Berlin/Heidelberg, Germany, 2012.

67. Wijaya, W.M.; Nakamura, Y. Predicting ship behavior navigating through heavily trafficked fairways by analyzing AIS data on apache HBase. In Proceedings of the IEEE 2013 First International Symposium on Computing and Networking, Matsuyama, Japan, 4-6 December 2013.

68. Yan, X.P.; Liu, C.G. Review and prospect for intelligent waterway transportation system. CAAI Trans. Intell. Syst. 2016, 11, 807-817.

69. Schilk, G.; Seemann, L. Use of its technologies for multimodal transport operations-River information services (RIS) transport logistics services. Procedia Soc. Behav. Sci. 2012, 48, 622-631. [CrossRef] 
70. Li, M.W.; Han, D.F.; Wang, W.L. Vessel traffic flow forecasting by RSVR with chaotic cloud simulated annealing genetic algorithm and KPCA. Neuralcompting 2015, 157, 243-255. [CrossRef]

71. Liu, J.X.; Zhang, T.; Liu, W. Research on combination forecasting method for ship traffic flow. Navig. China 2009, 32, 80-84.

72. Xu, W.X.; Chu, X.M.; Chen, X.Q. Methods of generating vessels for traffic flow simulation of bridge areas waterway. J. Syst. Simul. 2014, 26, 1644-1651.

73. Suo, H.; Wan, J.F. Security in the Internet of Things: A review. In Proceedings of the IEEE 2012 International Conference on Computer Science and Electronics Engineering, Hangzhou, China, 23-25 March 2012.

74. Li, S.C.; Tryfonas, T.; Li, H.L. The Internet of Things: A security point of view. Internet Res. 2016, 26, 337-359. [CrossRef]

75. Zhou, S.L. The Coverage Control Schemes for Maritime Sensor Networks Based on Nodes Cooperative Scheduling. Master's Thesis, Wuhan University of Technology, Wuhan, China, 2014.

76. Yin, Y.; Gong, Y.; Geng, X.F. Research on the safety protection technology of larger ship-shore communication. Electron. Tech. Softw. Eng. 2014, 10, 98-99.

77. Dai, M.; Zhong, N. The technical analysis and thinking of network fusion in the IoV communication for the inland river in China. Transp. Constr. Manag. 2012, 7, 96-97.

78. Ye, N.; Wang, Z.-Q.; Lin, Q.; Wang, R.-C. A Method for Driving Route Predictions Based on Hidden Markov Model. Math. Probl. Eng. 2015, 2015, 1-12. [CrossRef]

79. Prinsloo, J. Accurate Vehicle Location System Using RFID, an Internet of Things Approach. Sensors 2016, 16, 825. [CrossRef] [PubMed]

80. Ye, N.; Wang, Z.-Q.; Zhang, Y.-Y.; Wang, R.-C. A Method of Vehicle Route Prediction Based on Social Network Analysis. J. Sens. 2015, 15, 1-10. [CrossRef]

81. Jin, X.; Shao, J.; Zhang, X.; An, W.; Malekian, R. Modeling of nonlinear system based on deep learning framework. Nonlinear Dyn. 2016, 84, 1327-1340. [CrossRef]

(C) 2017 by the authors. Licensee MDPI, Basel, Switzerland. This article is an open access article distributed under the terms and conditions of the Creative Commons Attribution (CC BY) license (http:/ / creativecommons.org/licenses/by/4.0/). 